\title{
Determinantes sociales de la salud influyentes en la complicación de pacientes hipertensos y diabéticos que padecen insuficiencia renal
}

Diana Sofía Baquero Pérez; Médico. Universidad del Magdalena. Santa Marta. Colombia. Hospital José Antonio Socarrás. Municipio de Manaure Balcón del Cesar.

Mora-Sánchez Maria del Pilar; Psicóloga. Universidad Antonio Nariño. Sede Bucaramanga. Colombia. Fondo de Pasivo Social. Santa Marta, Magdalena

Rada-Salinas Liseth; Enfermera Profesional. Universidad Metropolitana. Barranquilla. Colombia. Mi IPS. Fundación, Magdalena.

Jiménez-Barbosa Wilson Giovanni wilsong.jimenez@utadeo.edu.co; Odontólogo, Universidad Nacional de Colombia, Bogotá, Colombia. Magíster en Administración, doctor en Ciencias Sociales, Niñez y Juventud, Universidad de Manizales, Manizales, Colombia. Profesor titular de los posgrados del Área de Gestión de la Salud y la Seguridad Social, Universidad Jorge Tadeo Lozano, Bogotá, Colombia

Fecha de recepción: 01/06/2017 Fecha de corrección 04/03/2019

Fecha de aprobación 30/05/2019

Fecha de publicación 30/06/2019

\begin{abstract}
Resumen
Introducción: Los determinantes sociales de la salud aluden al impacto que tienen sobre la salud y la enfermedad en la población diversos componentes demográficos. Objetivo: identificar los determinantes sociales de salud que influyen en la complicación de pacientes hipertensos y diabéticos que padecen insuficiencia renal, Metodología: estudio de tipo descriptivo, transversal, con enfoque cuantitativo en una muestra de 86 pacientes. Resultados: en el componente Biología Humana, prevalecieron pacientes entre 41 y 85 años de edad predominantemente en la década 60-69 años, sexo masculino; raza mestiza, con ambas patologías y mayor incidencia hipertensos, IRC en nivel de riesgo III y alto frecuencia de estrés. En el componente socio-ambiental el ingreso económico y el nivel educativo fueron bajos repercutiendo en escasas oportunidades laborales, no se apreció que los sistemas de saneamiento básico e infraestructura de las viviendas tengan significación sobre las complicaciones presentadas. El componente Estilo de Vida es el que presenta la mayor frecuencia, no solo por las conductas de los pacientes sino por la influencia de las condiciones económicas y sociales, que repercuten en una dieta adecuada. El componente Procesos Administrativos de Salud presenta la frecuencia más baja y los usuarios manifiestan una buena satisfacción con la calidad de la atención de la institución. La frecuencia observada para los determinantes sociales fue para Estilo de Vida 53,49\%; Socio-Ambiente 18,60\%; Biología Humana $17,44 \%$ y Sistema de Salud 10,47\%. Conclusión. El estilo de vida es el determinante con mayor influencia y el Sistema de Salud es el determinante con menor influencia sobre las complicaciones en los pacientes hipertensos y diabéticos que presentan insuficiencia renal crónica.
\end{abstract}

Palabras claves: determinantes sociales de la salud, enfermedad crónica, hipertensión, diabetes mellitus, complicaciones, insuficiencia renal

Copyright (C) Facultad de Ciencias de la Salud de la Universidad Tecnológica de Pereira. 1995-2018. Todos los derechos reservados
Social determinants of health influencing the complication of hypertensive and diabetic patients suffering from renal failure

\begin{abstract}
Introduction: The social determinants of health allude to the impact of different demographic components on health and disease in the population. Objective. To identify the social determinants of health that influence the complication of hypertensive and diabetic patients suffering from renal failure. Methodology. A descriptive, crosssectional study with a quantitative approach in a sample of 86 patients. Results. In the Human Biology component, patients between 41 and 85 years of age prevailed predominantly in the 60-69 years, male gender; Mixed race, with both pathologies and higher incidence of hypertension, IRC at risk level III and high frequency of stress. In the socio-environmental component, economic income and educational level were low, with little employment opportunities, it was not appreciated that the basic sanitation and housing infrastructure systems had a significant impact on the complications presented. The Lifestyle component is the one that presents the most frequency, not only for the behaviors of the patients but also for the influence of the economic and social conditions, which affect an adequate diet. The Administrative Health Processes component presents the lowest frequency and the users show a good satisfaction with the quality of care of the institution. Conclusion. The frequency observed in this study for social determinants was for Lifestyle $53,49 \%$; SocioEnvironment 18,60\%; Human Biology 17,44\% and Health System $10,47 \%$.
\end{abstract}

Key words: social determinants of health, chronic disease, hypertension, diabetes mellitus, complications, renal insufficiency

Copyright (C) Facultad de Ciencias de la Salud de la Universidad Tecnológica de Pereira. 1995-2018. Todos los derechos reservados 


\section{Introducción}

El concepto de determinantes sociales de la salud emerge con fuerza desde hace dos décadas, ante el reconocimiento de las limitaciones de las intervenciones dirigidas a los riesgos individuales de enfermar, que no tomaban en cuenta el rol de la sociedad y aluden al impacto que tienen sobre la salud y la enfermedad en la población, diversos factores como, la edad, sexo, el grupo racial o étnico, la educación, la ocupación, el ingreso económico, la residencia urbana o rural y las condiciones de habitabilidad o equipamientos del lugar en el que se vive o trabaja, y la accesibilidad y atención de los servicios de salud [1].

Es así como surgen diferentes modelos que buscan comprender la relación entre los determinantes y afectación de la salud de los individuos, entre los que sobresale el Modelo de Mark Lalonde (1974), adoptado por la Organización Mundial de la Salud (OMS). Según este Modelo se puede decir, esquemáticamente, que las condiciones de salud dependen de cuatro conjuntos de factores o componentes que identifica como determinantes de la salud: la biología humana, el ambiente socio-económico y educativo, los estilos de vida y los servicios de salud.

Con base a este Modelo, la Organización Mundial de la Salud (OMS) [2] define los determinantes sociales de la salud como "las circunstancias en que las personas nacen, crecen, viven, trabajan y envejecen, incluido el sistema de salud, que permitan intervenir de manera preventiva en la detección oportuna y contribuir al control cuando ha sido diagnosticada la enfermedad, así como disminuir las complicaciones derivadas del estilo de vida, de la distribución del dinero y los recursos a nivel mundial, nacional y local, que depende a su vez de las politicas adoptadas. Los determinantes sociales de la salud explican la mayor parte de las inequidades sanitarias, esto es, de las diferencias injustas y evitables observadas en lo que respecta a la situación de la salud".

Posteriormente, en 1991, Dahlgren y Whitehead [3], adaptaron el Modelo de Lalonde, diagramando los determinantes de la salud como capas de influencia. En este Modelo ampliamente difundido, el centro está representado por el individuo y sus características no modificables como son: edad, sexo, y que se proyectan hacia los

$$
\mathrm{n}=\frac{\mathrm{N} \mathrm{Z}^{2}(\mathrm{pq})}{\mathrm{d}^{2}(\mathrm{~N}-1)+\left(\mathrm{Z}^{2}(\mathrm{pq})\right)}
$$

Donde:

$\mathrm{n}=$ tamaño de la muestra

$\mathrm{N}=$ tamaño de la población.

$Z=$ Nivel de confianza, elevado al cuadrado. $95 \%=1.96^{2}=3,8416$

$\mathrm{d}=$ nivel de precisión absoluta. Referido a la amplitud del intervalo de confianza deseado en la determinación del valor promedio elevado al cuadrado. $5 \%=0.5^{2}=0,25$

$\mathrm{p}=$ proporción aproximada del fenómeno en estudio en la población de referencia

$\mathrm{q}=$ proporción de la población de referencia que no presenta el fenómeno en estudio (1-p). La suma de la p y la q siempre debe dar 1 . Por ejemplo, si $\mathrm{p}=0-8, \mathrm{q}=0.2$

$\mathrm{n}=\frac{111^{*} 1.96^{2 *} 0.5(1-0.5)}{0.05^{2} *(111-1)+\left(\left(1.96^{2}\right)+0.5(1-0.5)\right.}$
$\mathrm{n}=\frac{111 * 3.8416 * 0.25}{0.0025 * 110+(3,8416(0.25))}$
$\mathrm{n}=\frac{111 * 3.8416 * 0.25}{0.0025 * 110+0.9604}$
$\mathrm{n}=\frac{106,6044}{1,2354}$

Las variables que constituyeron el estudio están sustentadas por los determinantes sociales de la salud que establece la Organización Mundial de la Salud, los cuales son: las características biológicas y psicológicas; factores socio-ambientales, estilo de vida, y los procesos administrativos de los sistemas de salud que puedan influir en la complicación con insuficiencia renal crónica en los pacientes con hipertensión y diabetes mellitus que asisten a Hospital José Antonio Socarrás en Manaure Balcón del Cesar.

Los criterios de inclusión fueron pacientes de ambos sexos, atendidos en consulta interna y nefrología del Hospital José Antonio Socarrás en Manaure Balcón del Cesar, que presentaban algún estadio de IRC concomitantes a hipertensión arterial y/o diabetes mellitus. Los criterios de exclusión fueron pacientes con insuficiencia renal crónica no relacionada con hipertensión arterial y/o diabetes mellitus, o asociada a otra patología crónica como enfermedad vascular, dislipemia o cáncer.

Las fuentes primarias fueron pacientes hipertensos y diabéticos con complicación de insuficiencia renal crónica y las fuentes secundarias fueron revisión de Historias Clínicas, Revisión bibliográfica, consultas en Internet.

Para la recolección de los datos se utilizó una Encuesta (ver anexo 1), con la técnica del cuestionario, que permitió el análisis de cada uno de los indicadores contemplados en las variables de estudio. El cuestionario constó de un total de 22 preguntas, cerradas de una o múltiple selección de respuesta, distribuidas según los Determinantes Sociales de la Salud propuestos por la Organización Mundial de la Salud.

Para el Componente Biología Humana se formularon 6 preguntas.; para el Componente Condiciones Socioeconómicas, Educativas y Ambientales 7 preguntas; para el Componente Estilo de Vida 2 preguntas y para el Componente Intervenciones médico-sanitarias 7 preguntas. En este estudio el procesamiento de los datos se realizó mediante el programa Excel de Microsoft. Los resultados se presentaron a través de cuadros y grafica de barra.

En este estudio el procesamiento de los datos se realizó mediante el programa de estadística descriptiva Excel de Microsoft, versión 2016. Los resultados tuvieron un nivel de confianza del $95 \%$ y se expresaron en medidas de tendencia central. 


\section{Resultados}

\section{Componente Biología Humana}

El primer componente de los determinantes sociales de la salud corresponde a la Biología Humana. Los 86 pacientes que participaron en esta investigación, se ubicaron entre los 41 y 85 años de edad, (promedio 60,6 años); $46(53,49 \%)$ hombres y $40(46,51 \%)$ mujeres. Con respecto a la distribución por patología crónica se encontraron pacientes solo diabéticos 15 (17,44\%); pacientes solo hipertensos 19 $(22,09 \%)$ y pacientes con ambas patologías crónicas 52 (60,47\%),

En cuanto a raza, prevaleció la mestiza con $69.77 \%(n=60)$, ya que este grupo étnico es el predominante en la región costa norte colombiana, donde se localiza el municipio de Manaure Balcón del Cesar, seguida por la blanca, $16.28 \%(\mathrm{n}=14)$ y la negra, $13.95 \%$ $(n=12)$.

De acuerdo al nivel de riesgo de IRC en los pacientes que solo presentan una de las dos patologías se comprobó que la diabetes mellitus está causando mayores complicaciones. Así se tiene que para 19 los pacientes con hipertensión arterial, en el Nivel I alcanzan el $57,89 \%(\mathrm{n}=11)$ contra el 40,00\% $(\mathrm{n}=6)$ de los 15 pacientes diabéticos; para el Nivel III, los pacientes con hipertensión llegan al $42,11 \%$ $(\mathrm{n}=8)$ versus el $6,67 \%(\mathrm{n}=1)$; pero para el Nivel IV, no se encontraron pacientes hipertensos mientras que este nivel de complicación está en el $53,33 \%(n=8)$ de los diabéticos, por tanto, esta patología tiene un mayor impacto negativo para complicaciones de IRC.

En el presente estudio se encontró que $73(84,88 \%)$ pacientes padece alguna manifestación de estrés siendo la ansiedad el trastorno que en mayor número se presenta en los pacientes con $33,72 \%(\mathrm{n}=29)$. El $15,12 \%(n=13)$ manifestó no presentar ningún tipo de trastorno estresante.

\section{Componente ambiente socio-económico y educativo}

Dentro de los factores del segundo componente Socio-Ambiental de los determinantes sociales de la salud, se encontró que el 100\% cuentan con afiliación al Régimen Subsidiado, lo cual les permite contar con los servicios ofrecidos en el Plan Obligatorio de Salud Subsidiado (POS-S), que acoge a las personas de estratos 1 y 2 , que no se encuentren afiliados al Régimen Contributivo. En ese sentido se encontró que el $50 \%(n=43)$ de los usuarios pertenecen al estrato 1 y el otro $50 \%(n=43)$ pertenecen al estrato 2 .

En lo referente al nivel educativo, sobresalen con el $32.58 \%(\mathrm{n}=28)$ los pacientes con un nivel educativo de primaria completa, seguido por primaria incompleta $25.58 \%(\mathrm{n}=22)$, secundaria incompleta $24.42 \%(n=21)$, no estudió $16.28 \%(n=14)$ y secundaria completa $1.16 \%(n=1)$; lo que evidencia el bajo nivel educativo de los pacientes.

Al revisar la ocupación de los usuarios se observa que el 41,86\% $(\mathrm{n}=36)$ perciben un ingreso seguro mensual por ser empleados $10,47 \%$ $(\mathrm{n}=9)$, pensionados $5.81 \%(\mathrm{n}=5)$ e independientes $25.58 \%(\mathrm{n}=22)$. El restante $58.14 \%(\mathrm{n}=50)$ no perciben ingresos por encontrarse desempleados $27.91 \%(n=24)$ o ser amas de casa $30.23 \%(n=26)$.

Con referencia al ingreso mensual familiar, el $83.72 \%(n=72)$ percibe un ingreso mensual por hogar de 1 salario mínimo vigente, mientras que el 16,28 (n=14) manifiesta percibir un ingreso por encima de un salario mínimo vigente.

En cuanto a la zona de residencia, se encontró que el $82.56 \%(\mathrm{n}=71)$ de los usuarios habitan dentro del casco urbano del municipio de Manaure Balcón del Cesar, mientras que el 17.44\% (n=15) lo hacen en zonas rurales.

Tabla 1. Factores medio ambientales de vivienda de los pacientes con hipertensión y diabetes que presentan insuficiencia renal. Hospital José Antonio Socarrás en Manaure Balcón del Cesar. Enero 2017.

\begin{tabular}{l|l|c|c}
\hline \multirow{2}{*}{ VARIABLE } & \multicolumn{1}{c|}{ INDICADOR } & FRECUENCIA & PORCENTAJE \\
\hline \multirow{4}{*}{$\begin{array}{l}\text { Servicios básicos con } \\
\text { que cuenta la vivienda }\end{array}$} & Agua potable & 85 & 98,84 \\
\cline { 2 - 4 } & Luz eléctrica & 71 & 82,56 \\
\cline { 2 - 4 } & Gas domiciliario & 71 & 82,56 \\
\cline { 2 - 4 } & Transporte público & 86 & 100,00 \\
\hline \multirow{3}{*}{$\begin{array}{l}\text { Condiciones de in- } \\
\text { fraestructura de la } \\
\text { vivienda }\end{array}$} & Piso de tierra & 7 & 8,14 \\
\cline { 2 - 4 } & Obra negra & 30 & 34,88 \\
\cline { 2 - 4 } & Techo de zinc & 44 & 51,16 \\
\cline { 2 - 4 } & Letrina & 36 & 41,86 \\
\hline
\end{tabular}

Las características del entorno de vivienda son importantes en los determinantes de la salud, especialmente en aquellas que no reúnen condiciones adecuadas de habitabilidad donde la deficiencia no solo está en la infraestructura de la vivienda, sino también en los servicios básicos de saneamiento básico con que cuente, como se observa en la tabla 1.

Con respecto al acceso al agua potable el $98,84(\mathrm{n}=85)$ cuenta con este servicio, de los cuales solo una vivienda rural provee agua de acequia. Para los servicios de luz eléctrica y gas domiciliario, la totalidad de las viviendas urbanas $(n=71)$ cuentan con ambos servicios y por el contrario ninguna de las viviendas rurales $(\mathrm{n}=15)$ accede a luz eléctrica ni gas domiciliario.

Referente a la infraestructura de las viviendas, el factor más importante en las deficiencias, es el todavía uso de letrinas en el $41,86 \%$ ( $n=36)$ de las viviendas, constituidas por 21 urbana y 15 rurales.

\section{Componente estilos de vida}

Tabla 2. Factores del estilo de vida de los pacientes con hipertensión y diabetes que presentan insuficiencia renal. Hospital José Antonio Socarrás en Manaure Balcón del Cesar. Enero 2017.

\begin{tabular}{|c|c|c|c|}
\hline VARIABLE & INDICADOR & FRECUENCIA & PORCENTAJE \\
\hline \multirow{4}{*}{$\begin{array}{l}\text { Hábitos } \\
\text { adictivos }\end{array}$} & $\begin{array}{l}\text { Consumo de } \\
\text { cigarrillo }\end{array}$ & 7 & 8,14 \\
\hline & $\begin{array}{l}\text { Consumo de } \\
\text { alcohol }\end{array}$ & 14 & 16,28 \\
\hline & $\begin{array}{l}\text { Consumo de } \\
\text { café }\end{array}$ & 64 & 74,42 \\
\hline & Ninguno & 14 & 16,28 \\
\hline
\end{tabular}

En la tabla 2 se presentan los resultados del Estilo de Vida el cual es el tercer componente, y el más importante, en los determinantes sociales de la salud para las enfermedades no trasmisibles, por cuanto a pesar de las estrategias para enfrentar sus factores de riesgo, como son actividad física, ingesta de sustancias nocivas, adherencia al tratamiento, con frecuencia se presenta un efecto inverso a lo esperado sobre este componente de estilo de vida.

Como se aprecia en la tabla, la ingesta de café $74.42 \%$ es el hábito más frecuente seguido del de alcohol con $16.28 \%$ y el del cigarrillo 
con $8.14 \%$; Se correlacionó que siete pacientes mantienen todos los hábitos adictivos: fuman, beben y toman café. Estos pacientes tienen en común que todos son hombres, están en Nivel III de riesgo de IRC, son desempleados, perciben un ingreso mensual de un SMLV, pertenecen a la zona de residencia urbana y su nivel educativo se ubica en primaria incompleta.

Las modificaciones en el estilo de vida ofrecen el potencial de prevenir la hipertensión, y son efectivas en la disminución de la presión arterial y pueden reducir otros factores de riesgo cardiovascular a un bajo costo y con riesgo mínimo. Los pacientes deben ser estimulados agresivamente para adoptar estas modificaciones en el estilo de vida, particularmente si tienen patologías concomitantes como la diabetes mellitus. Aun cuando las modificaciones en el estilo de vida solas no son adecuadas en controlar las patologías crónicas, pueden reducir el número y dosis de los medicamentos necesarios para el manejo de estas enfermedades y prevenir sus complicaciones.

Tabla 3. Adherencia al tratamiento de los pacientes con hipertensión y diabetes que presentan insuficiencia renal. Hospital José Antonio Socarras en el municipio de Manaure Balcón del Cesar. Enero 2017

\begin{tabular}{l|l|c|c}
\hline \multicolumn{1}{c|}{ VARIABLE } & \multicolumn{1}{c|}{ INDICADOR } & FRECUENCIA & PORCENTAJE \\
\hline \multirow{3}{*}{ Citas médicas } & Cumple & 86 & 100,00 \\
& No cumple & - & - \\
& Total & 86 & 100,00 \\
Dieta alimenticia & Cumple & 50 & 58,14 \\
& No cumple & 36 & 41,86 \\
Toma oportuna de & Total & 86 & 100,00 \\
medicamentos & Cumple & 73 & 84,88 \\
& No cumple & 13 & 15,12 \\
Ejercicio físico & Total & 86 & 100,00 \\
& Cumple & 50 & 58,14 \\
& No cumple & 36 & 41,86 \\
& Total & 86 & 100,00 \\
\hline
\end{tabular}

Otra variable que se incluye dentro del componente Estilo de Vida es la adherencia al tratamiento farmacológico y no farmacológico, como se aprecia en la tabla 3, que contempla la asistencia a citas médicas, toma oportuna de medicamentos, dieta apropiada y realización de actividad física.

Como se observa, el 100\% ( $\mathrm{n}=86)$ de los pacientes asiste a las citas médicas programadas; pero se encontró que solo el $58.14 \%(\mathrm{n}=50)$ cumple con una dieta adecuada para el control de su patología crónica. El incumplimiento del $41.86 \%(n=36)$ de los usuarios tiene dos causales. El primero está relacionado con el bajo ingreso económico que no les permite acceder a los alimentos recomendados; por lo cual existe una relación directa entre estas dos variables. El segundo tiene una relación cultural, donde los usuarios se niegan a abandonar sus costumbres gastronómicas, jugando aquí un papel importante la variable educativa, que les inhibe entender la necesidad de evitar alimentos perjudiciales, como combinar más de un carbohidrato o alto consumo de grasas y sodio.

Se correlacionó que 7 pacientes que no cumplen con la dieta ni el ejercicio físico tienen en común que son todas mujeres, amas de casa, con un Nivel de Riesgo I de IRC, ingreso de 1 SMLV, zona de residencia urbana y nivel educativo en primaria incompleta.

\section{Componente servicios de salud.}

El cuarto componente de los determinantes sociales de la salud se refiere a los Procesos Administrativos de los Sistemas de Salud. En este sentido, se encontró que la ESE cumple en el 100\% $(n=86)$ de los usuarios con los indicadores evaluados de citas médicas oportunas, análisis y procedimientos oportunos y entrega de medicamentos POS.

Tabla 4. Cumplimiento de los procesos de atención al paciente hipertenso y diabético complicado con insuficiencia renal crónica, usuario de los servicios de salud. Hospital José Antonio Socarras en el municipio de Manaure Balcón del Cesar. Enero 2017

\begin{tabular}{l|l|c|c}
\hline \multicolumn{1}{c|}{ VARIABLE } & INDICADOR & FRECUENCIA & PORCENTAJE \\
\hline \multirow{2}{*}{$\begin{array}{l}\text { Citas médicas } \\
\text { oportunas }\end{array}$} & Cumple & 86 & 100,00 \\
\cline { 2 - 4 } & No cumple & - & 0,00 \\
\cline { 2 - 4 } & Total & 86 & 100,00 \\
\hline \multirow{2}{*}{$\begin{array}{l}\text { Análisis y proce- } \\
\text { dimientos opor- } \\
\text { tunos }\end{array}$} & Cumple & 86 & 100,00 \\
\cline { 2 - 4 } & No cumple & - & 0,00 \\
\cline { 2 - 4 } $\begin{array}{l}\text { Entrega oportuna } \\
\text { de medicamentos }\end{array}$ & Total & 86 & 100,00 \\
\cline { 2 - 4 } POS & No cumple & 86 & 100,00 \\
\cline { 2 - 4 } & Total & - & 0,00 \\
\hline \multirow{2}{*}{$\begin{array}{l}\text { Entrega oportuna } \\
\text { de medicamentos }\end{array}$} & Cumple & 76 & 100,00 \\
\cline { 2 - 4 } \begin{tabular}{l} 
NO POS \\
\cline { 2 - 4 }
\end{tabular} & No cumple & 9 & 10,47 \\
\cline { 2 - 4 } & Total & 86 & 100,00 \\
\hline
\end{tabular}

Asimismo, también se evaluó la incidencia de los procesos administrativos de los sistemas de salud sobre la complicación con insuficiencia renal en los pacientes que padecen hipertensión y diabetes, usuarios de la Institución de Salud, objeto de estudio, que se muestra en la tabla 4.

El indicador donde se encontró deficiencia fue en la entrega oportuna de medicamentos NO POS, los cuales debido a los procesos administrativos burocráticos, pueden presentar una demora, que en algunos casos, va más allá de los 30 días, incidiendo directamente en el estado de salud de los pacientes, afectando al mismo tiempo la calidad del servicio institucional.

Igualmente, al medir la satisfacción del usuario con la calidad de atención recibida del personal médico se encontró un $95.35 \%(\mathrm{n}=82)$ de satisfacción; atención recibida de otros profesionales de la salud (enfermeras jefes, auxiliares y laboratorios) hay satisfacción en el 96.15\% (n-83) de los usuarios; y de los servicios administrativos no están satisfechos en el $10.47 \%(n=9)$. Este último resultado se encuentra directamente relacionado con el incumplimiento de los medicamentos NO POS.

Para finalizar, que en la frecuencia observada para cada uno de los componentes de los Determinantes Sociales de la Salud que influyen en la complicación de pacientes hipertensos y diabéticos que padecen insuficiencia renal, usuarios del Hospital José Antonio Socarrás en Manaure Balcón del Cesar, destacándose como el mayor determinante Estilo de Vida con el 53,49\%.

\section{Discusión}

La salud es resultante de la confluencia de diferentes determinantes, como son los factores individuales, económicos, sociales, medioambientales y culturales que convergen para determinar los perfiles de salud/enfermedad. Con frecuencia, los estudios realizados 
sobre la salud se enfatizan solo en los factores biológicos, en menor medida en los socioculturales y muchos dejan de lado los factores medioambientales e institucionales. Por ejemplo, como señala Rodríguez [7] "los factores culturales pueden ser un factor positivo de cambio o pueden ser un obstáculo para alcanzar la salud”.

La búsqueda de los determinantes sociales y su influencia en la salud aluden al impacto que tienen sobre la enfermedad en la población variables como el grupo racial o étnico, la riqueza, la educación, la ocupación, la residencia urbana o rural y las condiciones de habitabilidad o equipamientos del lugar en el que se vive o trabaja y las desigualdades que se generan como consecuencia de las diferencias de estos componentes en materia de salud, que pueden ser innecesarias, evitables e injustas.

Con respecto a los resultados sobre las características personales y su relación con los determinantes sociales de la salud que influyen en la complicación con insuficiencia renal crónica, al comparar con el estudio de O'Riordan, citado en Pinto, Idrovo, Tapias, Vera y cols. [8] "se reportó un mayor riesgo de desarrollo de enfermedad renal crónica en mujeres", contrario a la presente investigación donde prevalecieron los hombres.

En cuanto a la comorbilidad entre hipertensión arterial y diabetes mellitus, varios estudios, entre ellos el de Anaya-Orozco [9], señala que la hipertensión arterial es frecuente en los diabéticos, afectando el $20-60 \%$ de la población con diabetes mellitus", lo cual coincide con los resultados de este estudio.

Agrega Anaya-Orozco que es interesante la asociación que se observa en una cantidad considerable de pacientes que presentan ambas enfermedades, lo cual ha llegado a plantear a los investigadores la posibilidad de un nexo fisiopatológico que las ligue entre sí. La prevalencia de hipertensión en la población diabética es 1,5-3 veces superior que en no diabéticos.

Así mismo, la presente investigación no coincide con estudios como el de Molina, Simal, Martín, Bellido y cols., [10], el cual señala que los estadios 2-3 de IRC, aumenta con la edad, siendo la prevalencia mayor en mujeres con una tendencia de aparición en edades medias, persistiendo en edades mayores de 65 años. Con el método CockroftGault, casi la mitad de las mujeres mayores de 65 años tienen una IRC estadio 3 frente a un tercio de los varones, ya que los hombres son quienes presentan mayor nivel de riesgo de IRC, que se vincula al mismo tiempo con una menor adherencia al tratamiento y un deficiente estilo de vida.

Vinaccia y cols., [11] consideran que la mayoría de las enfermedades crónicas afecta aspectos importantes de la vida en las personas que la padecen. Uno de los principales cambios y quizás el que genera mayor deterioro es el aspecto emocional, ya que la persona se ve obligada necesariamente a un proceso de adaptación rápido para el cual pasa por diferentes etapas que suscitan una serie de emociones comúnmente negativas (angustia, depresión, ansiedad, miedo, etc.).

El estrés tiene una repercusión directa en las enfermedades cardiovasculares y metabólicas porque genera una serie de hormonas en el cuerpo humano llamadas catecolaminas, que también se conocen como las "hormonas del estrés". El incremento excesivo y prolongado de estas hormonas aumenta la demanda de oxígeno del organismo por lo que el corazón debe trabajar más rápido de lo que acostumbra, aumentando la frecuencia cardiaca, es decir, al aumentar la fuerza y el número de latidos del corazón, la sangre saldrá del mismo con una mayor presión, lo cual es el principio básico de la hipertensión arterial.
Otras de las respuestas del cuerpo al estrés es el aumento del azúcar en la sangre, recordando que la este aumento obedece a una reacción primitiva de "lucha o huida" en la que el ser humano se prepara para enfrentar algo que los amenaza o huir para la protección, por lo tanto los músculos necesitarán un mayor aporte de energía y oxígeno para poder funcionar de acuerdo a estas demandas, y la principal fuente de energía del cuerpo es la glucosa, por lo que el hígado se encarga de liberar una mayor cantidad y el páncreas a su vez libera una hormona llamada insulina que facilita que esta glucosa sea convertida en energía por las células del cuerpo, el motivo por el cual el estrés crónico es un factor que contribuye al desarrollo de diabetes mellitus.

Puede decirse que una respuesta médica a los cambios biológicos que acompañan al estrés podría consistir en controlarlos con tratamiento farmacológico. Sin embargo, la atención debe dirigirse a hacer frente a las causas de la mala salud enfocándose en los determinantes sociales que lo causan.

Por ejemplo, el estudio canadiense de D'Arcy [12] publicado por la Organización Mundial de la Salud, determinó que las personas desempleadas tienen significativamente más dificultades psicológicas, ansiedad y síntomas de depresión, días de discapacidad, limitación de actividades, problemas de salud, hospitalizaciones y visitas médicas que las personas empleadas.

Como señala el estudio de Bravo [13] para la Comisión Económica para América Latina y el Caribe (CEPAL), la mayoría de las variables sociales (estratificación, ocupación, ingreso económico) están relacionadas con el nivel educativo. Por ello, la importancia de la educación va más allá de los límites de un objetivo propio del desarrollo social para incluirse como un requisito fundamental para lograr un mayor crecimiento personal y equidad.

Es indudable que, el desempleo se asocia con un estado de salud deficiente. La Organización Mundial de la Salud mostró que los niveles altos de desempleo y la inestabilidad económica causan problemas de salud mental significativos y efectos adversos sobre la salud física afectando no solo al individuo sino a su entorno familiar y social.

Una de las razones para al anterior inequidad económica radica en el bajo nivel educativo que tienen estas personas. El nivel educativo se considera una de las variables más influyentes en la toma de decisiones sobre la salud de la población y en la adaptación de los pacientes a las medidas de autocuidado. A mayor nivel educativo mejor comprensión sobre las necesidades de adherencia al tratamiento farmacológico y no farmacológico. Igualmente influye en la capacidad de las personas para acceder a una mejor ocupación y, por ende, a un mayor ingreso económico que permita la satisfacción de las necesidades de los miembros de la familia o para aumentar sus capacidades.

De allí, que en lo que se refiere al componente social, el fenómeno de las desigualdades en salud está caracterizado, según Daponte [14], por su carácter injusto y evitable, además de su ubicuidad, consistencia, su enorme magnitud, su carácter gradual, su tendencia creciente, su persistencia, su carácter histórico y su carácter adaptativo, lo que indica que los grupos sociales mejor posicionados socialmente son los más beneficiados de las acciones sanitarias y sociales dirigidas a mejorar la salud.

Los resultados respecto a la frecuencia observada para cada uno de los componentes de los Determinantes Sociales de la Salud que influyen en la complicación de pacientes hipertensos y diabéticos que padecen insuficiencia renal de este estudio son muy semejantes con los resultados señalados en las investigaciones realizadas por Carvalho y cols., [15], donde la frecuencia observada en diversos estudios de estos determinantes ha sido para estilo de vida del $51 \%$, 
biología humana 20\%; campo ambiental 19\% y servicios de salud del $10 \%$.

En complemento, la Organización Mundial de la Salud sostiene que las instalaciones higiénicas de saneamiento son esenciales para la salud pública, y que la falta de instalaciones de saneamiento mejoradas, como inodoros con cisterna y letrinas cubiertas propende al desarrollo de enfermedades. También sostiene que nueve de cada diez personas que utilizan letrinas viven en zonas rurales, pero reconocen que ha aumentado el número de viviendas urbanas sin instalaciones higiénicas de saneamiento adecuadas, debido al crecimiento acelerado que no acompañado de la correspondiente expansión de las instalaciones de saneamiento.

La falla en lograr un control adecuado de la hipertensión y la diabetes, a menudo implica un problema de adherencia del paciente. Hay implicaciones de tipo socioeconómico y conductuales. Es sabido, que no basta únicamente con la sola información del problema, ya que por ejemplo, en Estados Unidos, a pesar del conocimiento universal de que el ejercicio es "bueno" y el tabaquismo es "malo", hay un incremento importante en la prevalencia de la obesidad y el consumo de cigarrillos. Debido a que la hipertensión usualmente progresa en forma silente, a los pacientes se les hace difícil entender por qué es peligrosa. Por otra parte, los efectos adversos de los medicamentos disminuyen la adherencia del paciente al tratamiento y éste falla. La complejidad del tratamiento también juega un papel importante, sobre todo en pacientes diabéticos hipertensos, ya que deben de tomar varios medicamentos a la vez.

El componente el Estilo de Vida, es el determinante social de salud que tiene mayor influencia en las complicaciones de IRC en los pacientes con hipertensión arterial y diabetes mellitus 2, por lo cual es necesario que se realicen intervenciones y estrategias más profundas de apoyo educativo por parte del personal de salud de la institución para que se logre un cambio significativo en el autocuidado estos pacientes, con base a lo sostenido por la Dra. Dorotea Orem [16], quien refiere que "todas las personas tienen el potencial para aprender y desarrollar habilidades de cómo cuidar de sí mismos y de otros; y el potencial para ser motivado para dicho cuidado. Para lograr el desarrollo de ese potencial, es necesario el apoyo educativo, para guiar a otro". Por tanto, los esfuerzos por mejorar la salud de los pacientes no deben concentrarse sólo en los servicios de salud, sino se requiere intervenir en los estilos de vida.

En el contexto de la biología humana, el estrés tiene una cuota significativa como factor predisponente en las complicaciones de estos pacientes. En el contexto socio-ambiental debido al bajo ingreso económico percibido por cuenta de un deficiente nivel educativo, que se genera una influencia directa sobre los estilos de vida, por lo cual no siempre son decisiones de carácter individual sino conductas influenciadas por las circunstancias y oportunidades definidas por el medio social en el que están incluidos. En el contexto del estilo de vida integra el conjunto de conductas y hábitos personales que rodean la vida de los seres humanos; que fundamentalmente está configurados por el mantenimiento de una alimentación equilibrada y suficiente; abstinencia, o moderación, en el consumo de sustancias nocivas, la adherencia a las indicaciones farmacológicas y la práctica de una actividad física regular.

No obstante, para la observancia de un estilo de vida saludable deben relacionarse otros determinantes sociales que pueden afectar, en mayor o menor grado, el Estilo de Vida, como son el ingreso económico, el nivel educativo, el entorno social y el sistema de salud, entre otros.

Ejemplo de lo anterior, tal como lo ha demostrado la evidencia, es que el desempleo que pertenece al componente socio-ambiental, ocasiona estrés que pertenece al componente de la biología humana, por otra parte, para una buena salud se debe reducir el bajo nivel educativo.

En el contexto de los procesos administrativos de los sistemas de salud, a pesar que la ESE muestra una atención de calidad hacia los usuarios, que se refleja en el grado de satisfacción que éstos manifiestan; por las Políticas Públicas en Salud que conllevan a una complicada tramitología en el requerimiento de medicamentos NO POS, por lo cual se viola el principio fundamental de la justicia y equidad, produciendo una repercusión en la salud y bienestar de los individuos.

El componente el Estilo de Vida, es el determinante social de salud que tiene mayor influencia en las complicaciones de IRC en los pacientes con hipertensión arterial y diabetes mellitus 2 , por lo cual es necesario que se realicen intervenciones y estrategias más profundas de apoyo educativo por parte del personal de salud de la institución para que se logre un cambio significativo en el autocuidado estos pacientes.

En el contexto de la biología humana, el estrés tiene una cuota significativa como factor predisponente en las complicaciones de estos pacientes. En el contexto socio-ambiental debido al bajo ingreso económico percibido por cuenta de un deficiente nivel educativo, que se genera una influencia directa sobre los estilos de vida, por lo cual no siempre son decisiones de carácter individual sino conductas influenciadas por las circunstancias y oportunidades definidas por el medio social en el que están incluidos.

En el contexto de los procesos administrativos de los sistemas de salud, a pesar que la ESE muestra una atención de calidad hacia los usuarios, que se refleja en el grado de satisfacción que éstos manifiestan; por las Políticas Públicas en Salud que conllevan a una complicada tramitología en el requerimiento de medicamentos NO POS, por lo cual se viola el principio fundamental de la justicia y equidad, produciendo una repercusión en la salud y bienestar de los individuos.

Conflictos de interés: Los autores son independientes y manifiestan no tener conflictos de interés.

\section{Agradecimientos}

En primer lugar al Hospital José Antonio Socarrás en Manaure Balcón del Cesar por su invaluable colaboración al permitirnos el acceso a la información y obtención de los datos. A nuestra familia y amigos, motores e impulsadores en la realización de este trabajo.

Reconocimiento especial al doctor Néstor Raúl Ramos Navas quien aportó, en una primera instancia, con sus amplios conocimientos, una invaluable asesoría y orientación en este trabajo.

Conflictos de intereses: Los autores declaramos que no presentamos conflictos de intereses.

\section{Referencias}

1. Organización Mundial de la Salud (OMS). Comisión sobre Determinantes Sociales de la Salud. Ginebra-Suiza. 2005. (Fecha de consulta 8 de agosto 2016). Disponible en: http:// www.who.int/social_determinants/strategy/QandAs/es/

2. Organización Mundial de la Salud (OMS). Determinantes sociales de la salud. Ginebra-Suiza: 2009 (Fecha de consulta 3 de agosto 2016). Disponible en: http://www.who.int/social_ determinants/es/ 
3. Whitehead, M. y Dahlgren, G. Concepts and Principles for Tackling Social Inequities in Health Levelling up (Parte I). Copenhague: World Health Organization Copenhague 1991 (Fecha de consulta 4 de septiembre 2016). Disponible en: http:// www.msssi.gob.es/profesionales/saludPublica/prevPromocion/ promocion/desigualdadSalud/docs/concepDesigual.pdf

4. Organización Mundial de la Salud (OMS). Comisión sobre Determinantes Sociales de la Salud. Ginebra-Suiza. 2005. (Fecha de consulta 8 de agosto 2016). Disponible en: http:// www.who.int/social_determinants/strategy/QandAs/es/

5. Organización Panamericana de la Salud. (OPS). Determinantes sociales de la salud: los hechos irrefutables. Política de salud para todos (Health for All): Objetivo 14 - Entornos para la promoción de la salud. Londres. Ed: Wilkinson R, Marmot M. 2014 p 1-29 (Fecha de consulta 8 de agosto 2016). Disponible en: http://www.bvsde.paho.org/bvsacd/cd90/1008WILdet.pdf

6. Marchionni M, Caporale J, Conconi A, Porto N. Enfermedades crónicas no transmisibles y sus factores de riesgo. BID Banco Interamericano de Desarrollo. Resumen de Políticas. Bs.As. 2011. (Fecha de consulta 8 de agosto 2016). Disponible en: https://publications.iadb.org/bitstream/handle/11319/1358/ Enfermedades $\% 20$ cronicas $\% 20$ no $\% 20$ transmisibles $\% 20$ y $\% 20$ factores $\% 20 \mathrm{de} \% 20$ riesgo $\% 20$ en $\% 20$ Argentina: $\% 20$ prevalencia $\% 20 y \% 20$ prevencion $\% 20$ PROTOCOL. pdf? sequence $=6$

7. Rodríguez, L. Factores Sociales y Culturales Determinantes en Salud: La cultura como una fuerza para incidir en cambios en políticas de salud sexual y reproductiva. 2015. Ecuador (Fecha de consulta 10 de febrero 2017) Disponible en: http://www. ossyr.org.ar/pdf/bibliografia/2.6.pdf

8. Pinto, R, Idrovo V, Tapias M, Vera A., Jiménez S, Carrizosa E. Incidencia de enfermedad renal crónica y factores de riesgo para su desarrollo en pacientes postrasplante hepático en el Hospital Universitario Fundación Santa Fe de Bogotá 20042008. Trabajo Original. 2011 (Fecha de consulta 8 de marzo 2017). Disponible en: http://www.scielo.org.co/pdf/rcg/v26n3/ v26n3a03.pdf

9. Anaya-Orozco, M. Hipertensión arterial y diabetes mellitus. Rev Costarricense e Ciencias Médicas, 2004 25(3-4), 65-71. (Fecha de consulta marzo 30, 2017). Disponible en: http:// www.scielo.sa.cr/scielo.php?script $=$ sci_arttext\&pid $=\mathrm{S} 0253$ $29482004000200007 \& \operatorname{lng}=$ en\&tlng=es.

10. Molina A, Simal F, Martín JC, Bellido J, Ardua D, Mena FJ, González I, y cols., Prevalencia de la enfermedad renal crónica leve y moderada en la población general. Estudio Hortega. Rev. Nefrología 2004; 24 (4): 329-37. (Fecha de consulta 8 de septiembre 2016). Disponible en: http://www. revistanefrologia.com/es-publicacion-nefrologia-articuloprevalenciaenfermedad-renal-cronica-leve-moderadapoblacion-general-estudio-hortega-X0211699504017182

11. Vinaccia S; Orozco L. Aspectos psicosociales asociados con la calidad de vida de personas con enfermedades crónicas.
Diversitas: Perspectivas en Psicología, vol. 1, núm. 2, juliodiciembre, 2005, pp. 125-137. Universidad Santo Tomás Bogotá, Colombia. (Fecha de consulta 5 septiembre 2016). Disponíble en http://pepsic.bvsalud.org/scielo.php?script=sci_ arttext\&pid=S179499982005000200002\&lng=pt\&nrm=iso.

12. Organización Mundial de la Salud (OMS). Programa Especial de Análisis de Salud. Iniciativa de Datos Básicos en Salud y Perfiles de País. Documento de trabajo. Ontario (Canadá) 2000 Disponible en: www2.paho.org/hq/index.php?option=com tabs\&view $=$ article\&id...e

13. Bravo Rosa. Condiciones de vida y desigualdad social. Una propuesta para la selección de indicadores. CEPAL. 2000. (Fecha de consulta el 18 de marzo 2007. Disponible en: repositorio.cepal.org/handle/11362/31595

14. Daponte Codina, A., Bolívar Muñoz, J., García Calvente, M M. Las desigualdades sociales en salud. Granada: Escuela Andaluza de Salud Pública. 2009

15. Carvalho A, Marchiori P. Carvalho AI e Buss P. Determinantes Sociais na Saúde, na Doença e na Intervenção. In: Giovanella L, Escorel S, Lobato L, Noronha JC (org.) Políticas e Sistema de Saúde no Brasil. Rio de Janeiro: Ed. Fiocruz, 2008, p.141-166 Traducido del portugués. (Fecha de consulta 4 de septiembre 2016). Disponible en: http://ins.gob.pe/repositorioaps/0/0/ eve/evento maestria/Determinantes $\% 20 \mathrm{de} \% 201 \mathrm{a} \% 20$ salud-Traducci\%C3\%B3n $\% 20 \mathrm{al} \% 20$ espa $\%$ C3\%B1 ol $\% 20$ REVISADA.pdf

16. Orem D. Nursing: Concepts of Practice. 2a Ed. St. Louis:Mac Graw-Hill Book Company, 1991:2203 